\title{
On the Irreducible Representations of Groups That Contain a Subgroup of Index 2
}

\author{
U. Mutze
}

Sektion Physik der Universität München, D-8000 München 2, Federal Republic of Germany

\begin{abstract}
The objects under consideration are a group $G$ containing a subgroup $N$ of index 2 and an irreducible multiplier representation $U$ of $G$ by semiunitary ( = unitary or antiunitary) operators on a complex Hilbert space of arbitrary dimension. It is assumed that $U(g)$ is unitary for all $g$ belonging to $N$. Then the following assertion is proved. The representation of $N$ that is obtained by restricting $U$ to $N$ is either irreducible or an orthogonal sum of two irreducible representations.
\end{abstract}

\section{Introduction}

Let us consider a group $G$, a subgroup $N \subset G$, and an irreducible representation of $G$. By restricting this representation to the subgroup $N$ we obtain a representation of $N$. Which representations of $N$ are obtained in this way? This paper is devoted to an answer to a special case of this question.

Our most specializing assumption concerns $N$ : the index of this subgroup is assumed to be 2 (recall that the index of $N$ is the number of distinct cosets $g N$, $g \in G$ ). Examples that are representative for the scope of this paper are the following. [11].

(i) $G$ is the full Poincare group and $N$ is the orthochronous Poincare group

(ii) $G$ is the orthochronous Poincare group and $N$ is the proper orthochronous Poincaré group.

(iii) Examples (i) and (ii) with the Poincare group replaced by the inhomogeneous Galilei group [3].

(iv) $G$ is the group that is formed by the semiunitary (= unitary or antiunitary) operators on a Hilbert space and $N$ is the subgroup of unitary operators.

(v) Consider a semiunitary multiplier representation $U$ of a group $G$; then the so-called unitary subgroup $\{g \in G: U(g)$ is unitary $\}$ is a subgroup of index 1 or 2 .

Our second assumption concerns the kind of irreducible representations to be considered. To cover all those representations of the groups in (i)-(iii) that occur in physics, we consider semiunitary multiplier representations. These representations (see for instance $[4,8]$; on a classification of the multipliers of 
$G$ in terms of those of $N$ see [12]) are well known to be the natural group representations in quantum theory if there are no super-selection rules.

Finally we assume that the representations under consideration (which may or may not involve antiunitary operators) are unitary as representations of $N$. No topological or measure theoretical properties of groups or representations will be required.

Considering representations on finite dimensional vector spaces over an arbitrary algebraically closed field, Clifford [5] has answered our introductional question for any finite index of $N$; in the case of index 2 his answer is: The abovementioned representations of $N$ are either irreducible or direct sums of two irreducible representations. The essential statement in the present paper is that this answer is correct also for semiunitary multiplier representations on Hilbert spaces of arbitrary dimension. Let us call this statement the 1-2-Lemma (the representations under consideration are asserted to contain 1 or 2 irreducibles). Compared with the above-mentioned result of Clifford, this lemma is more general in that it does not assume the representation to be finite dimensional and more special in that it assumes that the representation space is a complex Hilbert space the norm function of which is conserved by the representation operators.

As is carried out in $[7,9]$, the 1-2-Lemma, combined with some algebra that is essentially known from the finite dimensional case $[1,2,13]$, yields the solution of the following problem:

(vi) Construct the irreducible semiunitary multiplier representations of a group, supposing that the irreducible unitary multiplier representation of its subgroups of index 2 are known.

Unfortunately, the arguments given in [7] and [9] to prove the 1-2-Lemma itself are conclusive in the finite dimensional case only-see the remarks in Section 3. Thus, to prove the 1-2-Lemma correctly means to complete the proof of the results on (vi) given in [7] (without proof, these results may be found in [3], pp. 146-148) and [9].

From a physical point of view, the most interesting applications of the abovementioned solution of (vi), and hence of the 1-2-Lemma, is that it allows to construct and to classify completely the irreducible semiunitary multiplier representations of the full Poincare group $[7,10]$ and of the full inhomogeneous Galilei group [3] starting with the well-known unitary irreducible multiplier representations of the respective proper orthochronous groups. Since this way of adjoining discrete symmetries, such as space inversion and time inversion, uses no topological and measure theoretical properties of the groups, it is surely less technical than the alternative way of generalizing the Mackey theory of induced representations such that antiunitary representation operators are allowed $[8,10]$.

\section{Formulation of the Results}

Conventions. We will use the words Hilbert space, semilinear operator, semiunitary operator, and conjugation operator respectively in the sense of complex 
Hilbert space of arbitrary dimension, linear or antilinear operator, unitary or antiunitary operator, and antiunitary operator the square of which is the identity operator.

Definition. Let $S$ be a set of semilinear bounded operators on a Hilbert space $H$. Then a closed subspace $L$ of $H$ is said to be irreducible under $S$ if $L$ is invariant under $S$ and no closed subspace of $L$, except $L$ and $\{0\}$, has this property.

As a criterion for the irreducibility of $H$ under a set of semilinear operators we shall need

"Schur's Lemma". Let $S$ be a set of semilinear bounded operators on a Hilbert space $H$ and suppose that for any $B \in S$ some complex multiple of $B^{*}$ belongs to $S$ (this is particularly satisfied if $S=U(G)$, where $G$ is a group and $U$ is a semiunitary multiplier representation). Then, $H$ is irreducible under $S$ if and only if the real multiples of the identity operator are the only bounded selfadjoint operators that commute with $S$ (i.e. with all $B \in S$ ).

1-2-Lemma. Let $G$ be a group containing a subgroup $N$ of index 2. Further, let $U$ be a mapping from $G$ to the set of semiunitary operators on a Hilbert space $H$ and suppose that $U$ has the following properties:

(i) $U(g) U(h)=w(g, h) U(g h)$ for all $g, h \in G$, where $w(g, h)$ is a complex number;

(ii) $H$ is irreducible under $U(G)$;

(iii) $U(g)$ is unitary for all $g \in N$.

Then one of the following assertions (iv) and (v) holds.

(iv) $H$ is irreducible under $U(N)$.

(v) $H$ is the orthogonal sum of two closed subspaces

$H_{1}$ and $H_{2}$ that have the following properties:

(1) $U(g) H_{1}=H_{1}, U(g) H_{2}=H_{2}$ for all $g \in N$,

(2) $U(g) H_{1}=H_{2}, U(g) H_{2}=H_{1}$ for all $g \notin N$,

(3) $H_{1}$ and $H_{2}$ are irreducible under $U(N)$.

\section{Proofs}

Proof of "Schur's Lemma". Let $L$ be a closed subspace of $H$ and let $P$ be the selfadjoint projection operator associated with $L$. Obviously, $L$ is invariant under some $B \in S$ iff $P B P=B P$. Therefore, $L$ is invariant under $B$ and $B^{*}$ iff $P$ commutes with $B$. Thus $L$ is invariant under $S$ iff $P$ commutes with $S$. Therefore, the Lemma is proved if we have shown that a bounded selfadjoint operator $A$ commutes with $B \in S$ iff its spectral projections $\left(E_{t}\right)_{t \in R}$ do so. If $B$ is linear, this is a well-known fact. If $B$ is antilinear, we choose a conjugation operator $K$ that commutes with $A$ and with the spectral projections $E_{t}$ (the existence of $K$ is evident from that version of the spectral theorem which represents $A$ as a multiplication operator on a direct sum of $L^{2}$-spaces [6]). Then it is clear that the following statements are mutually equivalent: $B A=A B ; B K A=A B K ; B K E_{t}=E_{t} B K$ for all $t \in \mathbb{R}$ (note that $B K$ is linear); $B E_{t}=E_{t} B$ for all $t \in \mathbb{R}$. This completes the proof.

Proof of the 1-2-Lemma. If (iv) does not hold, there is a closed subspace $H_{1}$ of $H$ that is invariant under $U(g)$ for all $g \in N$. Since these $U(g)$ are unitary the 
orthocomplement $\mathrm{H}_{2}$ of $\mathrm{H}_{1}$ is also invariant under $U(N)$. Since any $U(g)$ is bijective, this proves (v.1). With respect to the decomposition $H=H_{1} \oplus H_{2}$, we will use a self-explanatory operator-matrix-notation; in particular we write $U(d)=\left(\begin{array}{ll}A & B \\ C & D\end{array}\right)$ for some $d \in G$ with $d \notin N\left(A, B, C, D\right.$ are bounded semilinear mappings $H_{1} \rightarrow H_{1}$, $H_{2} \rightarrow H_{1}, H_{1} \rightarrow H_{2}, H_{2} \rightarrow H_{2}$ ). By virtue of (v.1), any operator $U(g)$ with $g \in N$ is diagonal in this notation. In order to prove (v.2), it is obviously sufficient to show that $U(d)$ is skew-diagonal i.e. $A=0$ and $D=0$. We show first that the linear bounded operator $Z:=W U(d)^{*}$ with $W:=\left(\begin{array}{rr}A & 0 \\ 0 & -D\end{array}\right)$ is selfadjoint and commutes with $U(G)$. As is easily seen, any of the following statements implies its follower: $U(d) U(d)^{*}=1 ; A C^{*}=-B D^{*} ; W U(d)^{*}=U(d) W^{*} ; Z=Z^{*}$. Thus $Z$ is selfadjoint. To show that $Z$ commutes with $U(G)$, it is obviously sufficient to show that it commutes with $U(d)$ and $U(N)$. Let us begin with $U(d)$. Since we have $\mathrm{U}(d)^{2}=w(d, d) U\left(d^{2}\right)$ and $d^{2} \in N$, the operator $U(d)^{2}$ is diagonal so that $A B=-B D$ and $C A=-D C$ and hence $W U(d)=U(d) W$ and $Z U(d)=U(d) Z$. Now we consider the condition $Z U(g)=U(g) Z$ for $g \in N$. It is clearly equivalent to $W V(g)=U(g) W$ with $V(g):=U(d)^{*} U(g) U(d)$. $V(g)$ is diagonal since it is a complex multiple of $U\left(d^{-1} g d\right)$ and $d^{-1} g d$, as $g$, belongs to the invariant subgroup $N$ (a subgroup of index 2 is always an invariant one). Let us write $V_{1}(g), V_{2}(g)$, and $U_{1}(g), U_{2}(g)$ for the diagonal elements of $V(g)$ and $U(g)$. Then the condition

(a) $X V(g)=U(g) X$ for $g \in N$ and any bounded semilinear operator $X=\left(\begin{array}{ll}Q & R \\ S & T\end{array}\right)$ is obviously equivalent to the following system of equations

$$
Q V_{1}(g)=U_{1}(g) Q, T V_{2}(g)=U_{2}(g) T,
$$

(b)

$$
R V_{2}(g)=U_{1}(g) R, S V_{1}(g)=U_{2}(g) S .
$$

Now it is evident from the definition of $V$ that $X=U(d)$ is a solution of (a) and hence that $Q=A, R=B, S=C, T=D$ is a solution of (b). Then, $Q=A, R=0$, $S=0, T=-D$ is also a solution of (b) so that $X=W$ is a solution of (a). Thus we have shown that the selfadjoint operator $Z$ commutes with $U(G)$. Since $H$ is irreducible under $U(G)$, "Schur's Lemma" shows that $Z$ is a real multiple of the identity operator, say $Z=z 1$. This is equivalent to $W=z U(d)$ and hence to the equations $A=z A, 0=z B, 0=z C,-D=z D$. If $z \neq 0$ we conclude $B=0$ and $C=0$, as a consequence of which the spaces $H_{1}$ and $H_{2}$ were invariant under $U(d)$ and hence under $U(G)$ in contradiction to (ii). Thus we have $z=0$ and hence $A=0, D=0$, which completes the proof of (v.2). Now assume that (v.3) does not hold. Then $H_{1}$ or $H_{2}$ contains a non-trivial closed subspace $L$ that is invariant under $U(N)$. By (v.2), $U(d) L$ is orthogonal to $L$ and, therefore, $L \oplus U(d) L$ is a closed non-trivial subspace of $H$, which, by (v.1) and (v.2), is easily seen to be invariant under $U(G)$ in contradiction to (ii). This completes the proof.

Remarks. The main statement of the present Lemma is used in [9] without proof: that there is a closed subspace of $H$ that is irreducible under $U(N)$. In the 
last step of our proof it is essential that $U(d) L$ is orthogonal to $L$; if we know only $L \cap U(d) L=\{0\}$ it is not clear that $L+U(d) L$ is closed. Therefore, the irreducibility of $H$ under $U(G)$ has no direct bearing on this space. This point is overlooked in both [7] and [9].

Acknowledgements. I am indepted to Dr. R. H. Brennich for a suggestion that allowed to simplify considerably an earlier version of the main proof. Further, I am grateful to Professor H. J. Meister and Dr. D. Castrigiano for many useful discussions.

\section{References}

1. Boerner, H. : Darstellungen von Gruppen, p. 100. Berlin-Heidelberg-New York: Springer 1967

2. Bradley, C. J., Davies, B. L. : Rev. Mod. Phys. 40, 359-379(1968)

3. Brennich, R. H. : Ann. Inst. Henri Poincaré 13, 137-161 (1970)

4. Cattaneo, U. : Rept. Math. Phys. 9, 31-53 (1976)

5. Clifford, A. H. : Ann. Math. 38, 535-550 (1937)

6. Dunford, N., Schwartz, J. T. : Linear operators, Vol. II, p. 1209. New York: Interscience Publishers 1963

7. Mutze, U. :Doctoral dissertation, München (1971)

8. Parthasarathy, K. R. : Commun. math. Phys. 15, 305-328 (1969)

9. Shaw, R., Lever, J. : Commun. math. Phys. 38, 257-277 (1974)

10. Shaw, R., Lever, J. : Commun. math. Phys. 38, 279-297 (1974)

11. Streater, R. F., Wightman, A. S. :PCT, spin and statistics, and all that, pp. 10, 14. New York: Benjamin 1964

12. Van den Broek, P. M.: Rept. Math. Phys. 9, 321-330 (1976)

13. Wigner, E. P. : Group theory, pp. 340-344. New York: Academic Press 1959

Communicated by H. Araki

Received November 1, 1977 
